\title{
Prediction of Air pollution in Al-Hmadi City using Artificial Neural Network (ANN)
}

\author{
Al-Rashed, A. ${ }^{1 *}$, Al-Mutairi, N. $^{2}$ and Boureslli A. ${ }^{3}$ \\ ${ }^{1}$ Science department, public Authority of Applied Education and Training, Kuwait. Email: ahmedbufarsan@gmail.com \\ ${ }^{2}$ Department of Civil Engineering, Kuwait University, P.O.Box 5969, 13060, Safat Kuwait \\ ${ }^{3}$ Science department, public Authority of Applied Education and Training, Kuwait
}

Received: 11/04/2020

Accepted: 27/08/2020

Published: 20/12/2020

\begin{abstract}
Neural networks application (ANNs) has been used at recent years in several technology areas. Applying artificial neural networks (ANN) has too many environmental engineering problems provide remarkable success. The objective of this project is to predict and show the accuracy of using neural network in air quality prediction in Al Ahmadi city. Several parameters such as carbon dioxide $\left(\mathrm{CO}_{2}\right)$, ammonia, benzene, relative humidity, temperature, air velocity and air direction are used in this study, for period of four years. The correlation coefficient was used to indicate the performance of ANN. The networks provide a good prediction performance with $\mathrm{R}$ value 0.93308 for $\mathrm{CO}_{2}, 0.81805$ and 0.923 for Ammonia and Benzene, respectively.
\end{abstract}

Keywords: Neural Network, Forecast, Air pollution, Quality

\section{Introduction}

The public health has been impacted by air smog for long time. Natural and man-made actions cause Harmful chemicals emission to the environment which have great influence on health and environment. In the last few years burning fuel accelerated and become the main effect on changing air quality. Poor air quality in cities is associated with rapid rate of great deliberation of vehicular consume fuels. Air pollution increased regularly and in vigorous and spread in vast geographical locations. Know a day's industry considered vital reason causing damage for the environment and air quality particularly. Carbon monoxide, sulfur dioxide, Nitrogen oxide, ozone and other air pollutants with different chemical properties are in great effect to the environment either in a short or long term. Diseases such, respiratory cancer and heart attack resulted from gases spread in the open air [1] and [2]. According to the World Health Organization statistics, more than two million people die every year. The main defendant for such high death numbers is simply different gases emitted from factories with all aspect of industrial activates. Percentage wise more than from car accidents. In conclusion, Human must implement the essential arrangements to reduce the pollution caused by their harmful waste. As addition air pollution has aggressive effect in people wellbeing correspondingly other sides of the environment like optical potentials, plant life, animals, lands and water feature [3]. The main factors that should take in consideration to determine the level of air pollution are the physical and chemical properties of pollutants, topography and weather conditions such as wind, temperature, air turbulence and rainfall. The emissions of gases form electrical power plants in Kuwait refineries, gases emission from exhausts of cars, are major sources of pollution, [4-6].

Kuwait Environmental Public Authority did a study between March 2011 and February 2012 for Ahmadi area on $\mathrm{NO}_{2}$ and $\mathrm{SO}_{2}$ concentrations concluded that winter had higher $\mathrm{NO}_{2}$ and $\mathrm{SO}_{2}$ readings than in the summer. According to the study the source of nitrogen oxides was the motor vehicles and the source of sulfur dioxide was different industrial sources. Artificial neural networks has an ability in forecasting unclear data and effective use of this approach in different domains encourage of using ANN to forecast air feature depend on an ancient data.one of the proposes of The study is the usage of neural network over years to predict air quality. Quality Forecasting - also referred to sometimes 'Atmospheric Diffusion Modeling. Data- is simulated to predict how air pollutants disband in the atmosphere. The simulation provides result represent concentration for each air pollutants in air of the chosen region. Experts develop different models of prediction in many institutions and research labs around the world successfully use one or several of them to realize the real-time forecast simulation and effectiveness. The main purpose of many studies is to find air quality prophets based in neural networks to handle the narrow amount of data groups and be durable for dealing with records with faults and noise. The use of neural networks has expended over the years in several technology areas. Implementation of Artificial neural network to many problems in environmental domain have achieved satisfied results. Advanced models were

Corresponding author: Al-Rashed, A., Science department, public Authority of Applied Education and Training, Kuwait. Email: ahmedbufarsan@gmail.com 
introduced for prediction based on annual and daily data. These simulations could forecast the quality of air with conservative reliability.

\section{Al-Ahmadi city}

Al-Ahmadi city is located in the southeastern of Kuwait (Fig. 1) with an area of $5,120 \mathrm{~km}^{2}$,apopulation of $(394,000)$ heavily polluted city due to rapid increase in vehicles and industry. Such as oil refiners, causing sufficient effect in air quality there. Kuwait Institute for Scientific Research involved in different studies and projects to monitor and evaluate air quality in hospitals, factories and oil -fields in the area and it relation to urban growth, such growth increase the demand on different resources (electricity and water). Dust, gases and other manufacturing pollutants resulted in a sharp increase in a cancer among al-Ahmadi residents (Table 1, Figure 2).

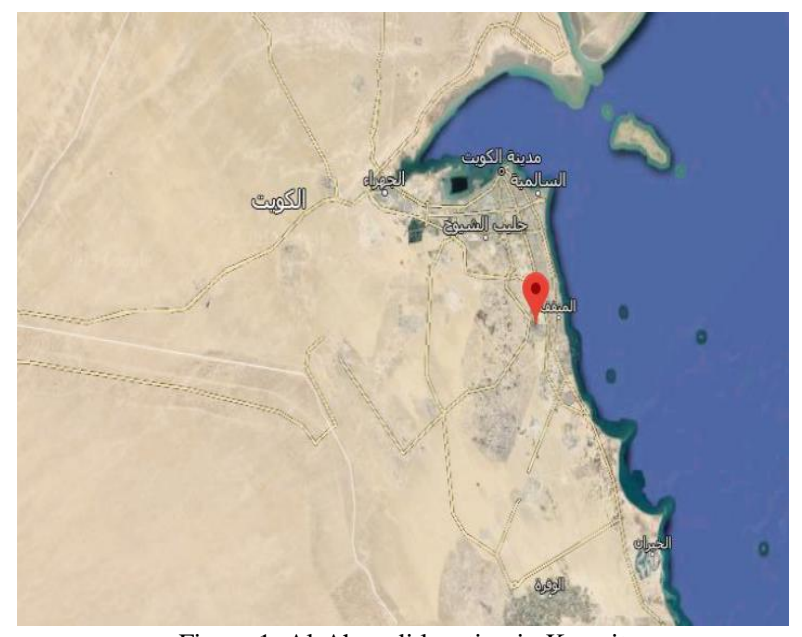

Figure 1: Al-Ahmadi location in Kuwait

\section{ANN modeling approach}

The ANN designs have many benefits over oldstyle semi-empirical simulations, although without a theory, it is essential to identify the information cluster [7]. This displays data about processing and is capable of enhancing the depiction of parameters of input and output. Using match inputs, this picture can be used to forecast results [8]. The dual-layer ANN predicted just about every measurable feature between results and input vectors by selecting a suitable weight related group and transferring any features [9]. This has interrelated 'node ' or 'neuron' layers as shown in Fig.

\subsection{ANN Modeling Method}

The method arrange procedure based on six serial stages: (i) choosing of ideal ANN-based model manner; (ii) choosing stimulation factor; (iii) selecting the best parameters for teaching, training rate and impulse frequency; (v) weights of initialization and network preferences; (vi) testing as well as analysis of the model; and (vii) model estimation.

\subsubsection{Ideal ANN Model Manner Choosing}

Amount with variables initially equivalent to an amount for neurons of the initial layer (i.e., variables are weather and pollutant concentrations in the current effort). The resulting layer includes single neuron, i.e. contaminant quantity. The resulting layer includes single neuron, i.e. contaminant quantity. Amount in neuronal in a secret coating is measured by a number for designs and a quantity for product / input neurons, a volume with sound measurements, a type of system, a kind for stimulation reaction was applied in a secret coating [10]. One concealed layer was capable of estimating several nonlinear functions as well as levels of ins and outs [11]. By training, in a hidden layer we can get the number, many networks and valuing the consistent faults of the test group data in the unseen sheet of few neurons and yield great testing errors because of under-fitting and mathematical nuances. As conflicting, many hidden layer neurons chief little training fault, however high testing error, because of through convenient and great variance [12].

\subsubsection{Stimulation Function}

A function is needed in network to build non-linear connection concerning input and output factor, to be able to attach and relate the factors [12]. In research for [7] and [9], articles related to poor air quality were discussed confirming which the hyperbolic sigmoid activation function seems to be faster and efficient than logistic sigmoid activation function to represent the nonlinearity between the hidden layer neurons [13]. Build on the hyperbolic tangent feature for hidden neuronal layers for this research. In addition, the ' individuality feature ' was used for their specific purpose results in the entry and output layer cells [9].

\subsubsection{Factor in Learning}

Training is carried out on the multilayer neural network. Preparation requires a custom information series comprising input and connected production. Back propagation neural network seems to be highly useful for training in the multi-layer $\mathrm{NN}$ [14]. In the back-propagation training, $\eta$ and $\mu$ are utilize to 'fast' or 'slow down' the interchange the mistakes [15]. Its learning scheme for back-propagation offers an "assessment" of the heavy space route calculated by a Gradient Descent Technique [16]. Significant lowering of the ' $\eta$ ' rate effects in small changes in the weight of the synapse from one repeat to the next and reduces the speed of training. However, its rise in the ' $\eta$ ' speed increases the speed of practice of a system because of big differences throughout neuronal measurements and therefore creates a system when unbalanced. In backpropagation teaching algorithm, the word ' $\mu$ ' was adapted in preventing system disturbance. The range of values ' $\pi$ ' and ' $\mu$ ' ranges from 0 to 1 [17] and [13].

\subsubsection{Original Weights of the Network}

Before beginning the instruction, the weights of neural networks and free parameters are crucial. The network's main weight and prejudice values help the learning processes to converge rapidly. Throughout the present research, both network distortion variables are laid to random amounts equally distributed with the distance $-2.4 / \mathrm{Fi}$ to $+2.4 / \mathrm{Fi}$, where Fi has been complete amount in outputs. A slightest variety of allocation reduces the likelihood of neuron saturation of a system to thus eliminating any mistakes happening [18].

\subsubsection{Practice and Monitoring}

Its 'supervised' learning algorithm is mostly used to trained the network. It is skillful by producing identified output and input figures in a well-arranged method toward system [17]. Learning includes finding a number of network weights so that the system can depict the basic models in the exercise information. It is achieved by reducing its design mistake to all models of input and linked result [7]. Its network ' setups ' ' in teaching ' a coaching algorithm in ' over-training ' and ' local ' minima outcomes in mistakes of elevated design forecast [9]. 


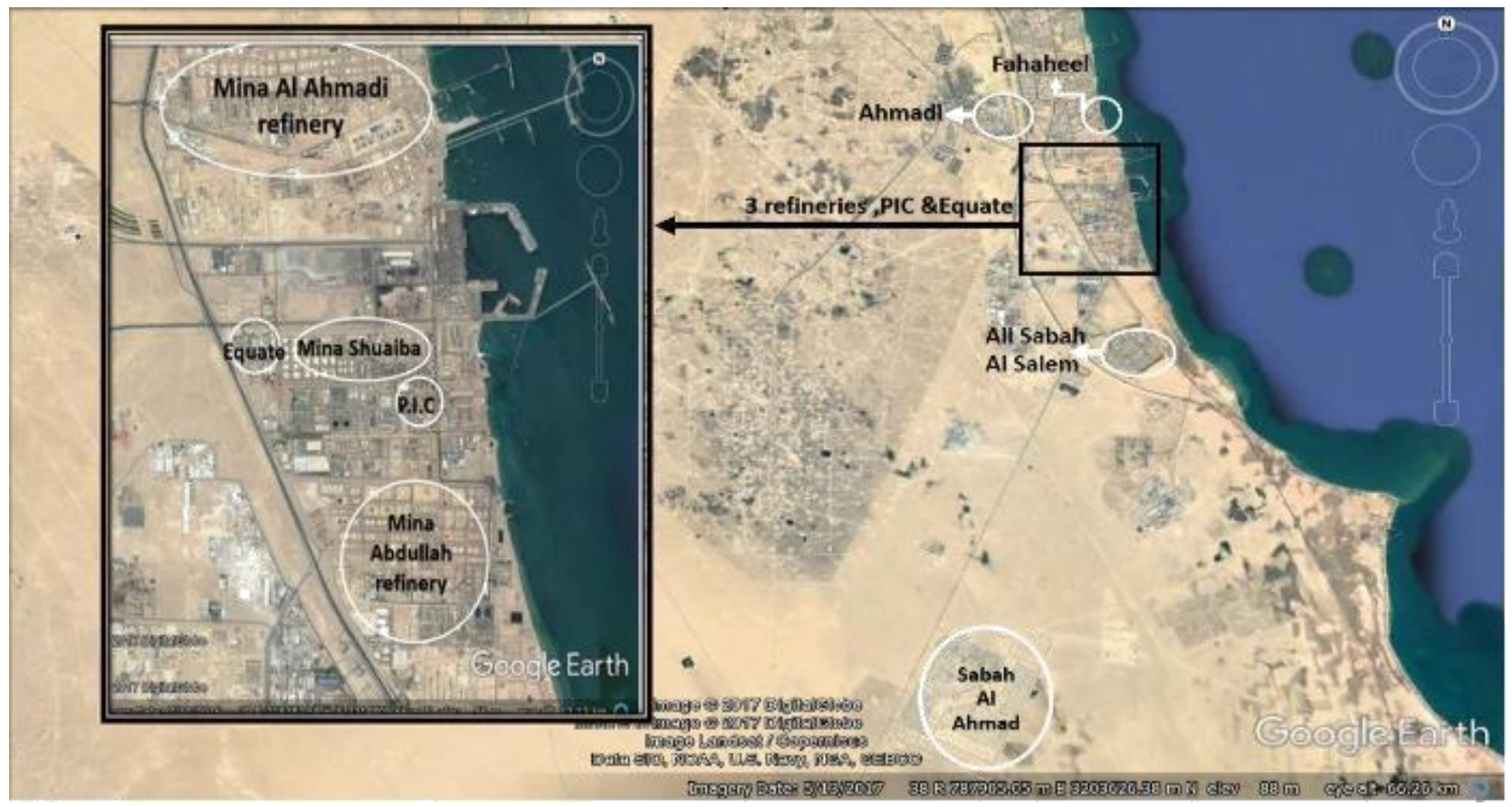

Figure 2: Location map of refineries in Alahmadi city

Table 1: some of industrial facilities in Ahmadi area.

\begin{tabular}{lll}
\hline Petrochemical facility & The processing capacity & Number of Stacks \\
\hline Mina Al-Ahmadi (MAA) & $466,000 \mathrm{bpd}$ & 65 \\
Shuiba (SHU) & $200,000 \mathrm{bpd}$ & 39 \\
Mina Abdullah (MAB) & $270,000 \mathrm{bpd}$ & 47 \\
Kuwait Petrochemical & The production capacity of urea is 1,040,000 TPA & Ammonia plant \\
Industries Company (PIC) & The production capacity of ammonia is 620,000 TPA & includes 10 stacks \\
EQUATE Petrochemical Company & The annual production of this company is over 6 million tons & 16 \\
\hline
\end{tabular}

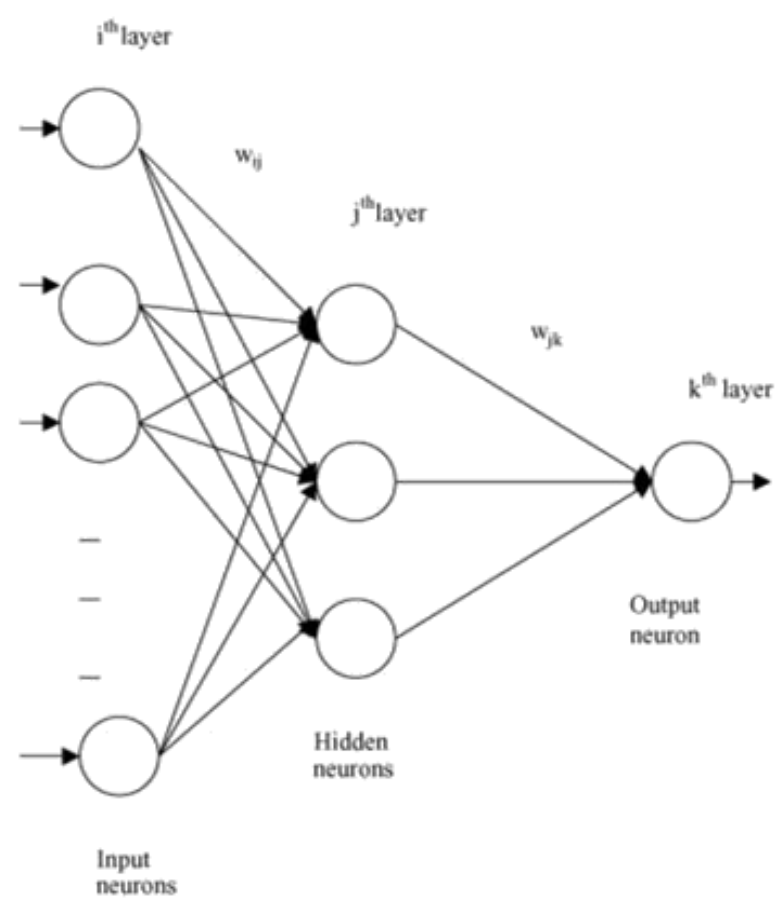

Figure 3: Common control system neural network construction
Too much training maybe prevented with testing a system with subgroup of production/entry to specify measurements with testing the rest of sample information and check the design predictions precision [19]. As a consequence, it is definitely the amount of 11. Learning algorithm for back-propagation has been the best in modeling research of air pollution [7]. The method separates all information to 3 sections: ' teaching information, ' and ' test information set ' and the ' assessment information set. Most of measurements used for training target is formulated in the ' training data set; ' and ' test data set ' for checking Simplification in NN system taught. Its learning remains at a standstill while 'sample information set' introduction leads to the smallest design mistake. Finally, the design is certified using the ' assessment information collection (Dorling and Gardner, 1998). The back-propagation learning algorithm's step-by-step method as shown below. The entry is multiply by a random main weight and add the outcome as follows:

$P j=h / j=i \sum W I j(x i+b j) ; i=1,2, \ldots, n \quad ; j=1,2, \ldots, H=1$ (1)

(1) where $P j$ is the input to the ' $j$ ' hidden layer neuron, xi the numerical value of the (i) input layer neuron, (wi) the weight of the (i) input layer neuron to ( $j$ ) the hidden layer neuron, $n$ is the number of the input layer neurons, $H$ the number of the hidden layer neurons and (bj) is the favoritism value for the (j) th hidden layer neuron. (ii) Change the hidden layer output by a 
sigmoid transfer function $\mathrm{f}(\mathrm{P} \mathrm{j})$. (a) Logistic function:(b) Q1= $1,2 / 1+e-p j, j=1,2, \ldots$, H. (c) (b) hyperbolic tangent. Where $Q(j)$ is the output of the neuron with a hidden layer ' $\mathrm{j}$ '. (iii) Multiply the hidden output layer weight by the hidden layer outputs and sum as:

$R K=h / j i \sum W j K Q j+B k \quad K=I j ;=1,2, \ldots, H$

where $\mathrm{R} \mathrm{k}$ is the input to the (k) output layer neuron, $\mathrm{w} \mathrm{j} \mathrm{k}$ the weight of the (j) the hidden layer neuron to the k output layer neuron, $\mathrm{m}$ the number of the output layer neuron and $\mathrm{b} \mathrm{k}$ is the preference value for the $\mathrm{k}$ output layer neuron. (i) Convert the output, R $\mathrm{k}$ by the transferal function to achieve the network out puts (YK). (ii) The network outputs are then related with observed values, and an error at the $\mathrm{k}$ output neuron is calculated: $\mathrm{EK}=\mathrm{Ti}-\mathrm{Y} \mathrm{k}$. Where $\mathrm{T} \mathrm{k}$ is the practice (real) value. The general standard used in the back-propagation learning method is the 'delta rule', which is founded on the decreasing the sum of squares of the error obtained in Equation.

\section{Objectives}

The objectives of this study are: (a) to predict concentrations of Benzene, $\mathrm{CO}_{2}$ and Ammonia in Ahmadi; (b) to analyze the predicted concentrations of pollutants; and (c) to compare the predicted concentrations with the standard limits set by Kuwait Environment Public concentrations of pollutants within the study area domain.

\section{Materials and Methods \\ 5.1 Data Sets}

In this study measurements involved includes daily outside temperature, relative humidity, air pressure, wind direction and speed, and daily concentration of $\mathrm{CO}_{2}$, ammonia and benzene in $\mathrm{Al}$ Ahmadi during 4 years period from 2014 to 2017. The data were delivered by (DGCA) Department of Meteorology. Measurements have been distributed in two sets, with artificial neural network learning as well as evaluating are validating its effectiveness and accuracy.

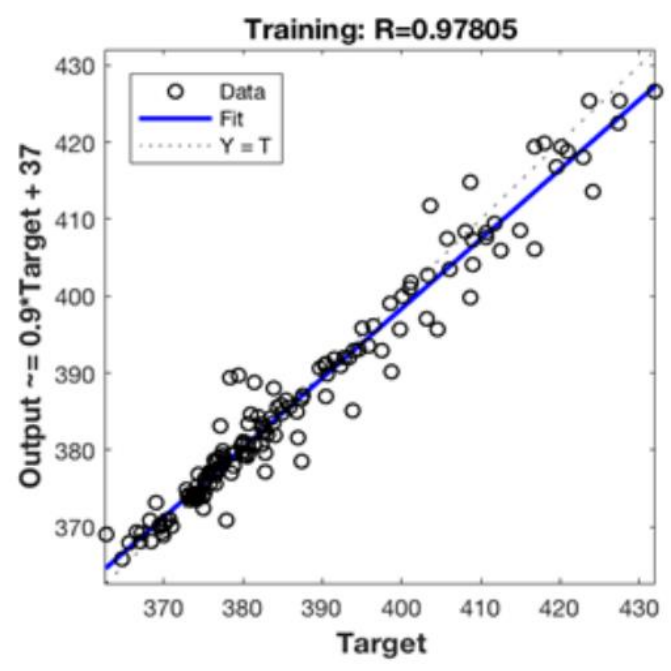

Figure 4: Correlation coefficient $\mathrm{R}$ of $\mathrm{CO}_{2}$ for validation

\subsection{Software}

MATLAB NN has been applied as adaptable as well as easy start to improve quality of the air forecasting system. Range data with NN enhancement is easily selected from the NN Toolbox proposal.

\subsection{Air Quality Prediction Using ANN Model}

As network construction, a 3-layer perceptron model was used. Initial entry coat comprises the entry data from network. In the input layer there were seven neurons as well as three contaminants which is $\mathrm{CO}_{2}$, ammonia and benzene four variables which is relative humidity, temperature, air pressure, and wind velocity. Amount with secret layers the limitations to be selected in the model are the quantity for neurons for a secret layer. Therefore, to enhance Artificial Neural Network efficiency, one or two hidden layers as well as varying neuronal measures have been selected. Final surface was production surface that is the estimation of template focus. $\mathrm{CO}_{2}$, ammonia and benzene were used as the variables in the output. Sooner than usual end, the registry has been separated in to three parts. Fifty percent of information have been used for system teaching, twenty-five percent for verification, as well as the residual twenty-five percent for network testing. Correlation coefficient $\mathrm{R}$ has been selected for numerical parameters for calculating system efficiency.

\section{Results and Discussion \\ 6.1 ANN Modeling}

In this study Feed-forward neural network was applied. The number in the hidden layer were 21 . The biases and weights were changed due to gradient-descent back-propagation in the training phase. The correlation coefficient $\mathrm{R}$ which is statistical measure was chosen for determining of the network efficiency. The training, validation and test for $\mathrm{CO}_{2}$ in fig. 4, 5, 6 and 7. While the correlation coefficient $\mathrm{R}$ of Ammonia shown in figures $8,9,10$ and 11 . The correlation coefficient $\mathrm{R}$ of benzene shown in figures $(12,13,14$ and 15$)$.

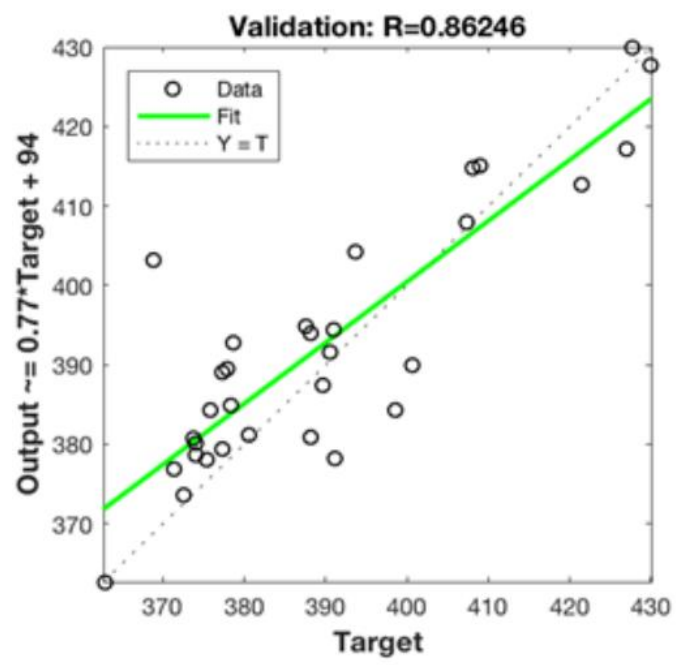

Figure 5: Correlation coefficient $\mathrm{R}$ of $\mathrm{CO}_{2}$ for training 




Figure 7: Correlation coefficient $\mathrm{R}$ of $\mathrm{CO}_{2}$ for test



Figure 8: Correlation coefficient $\mathrm{R}$ for all

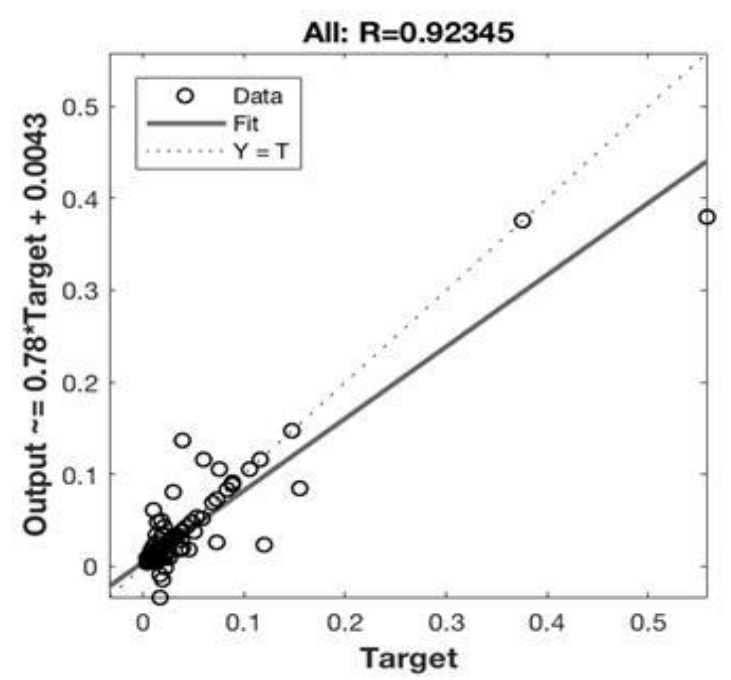

Figure 10: Correlation coefficient $\mathrm{R}$ of ammonia for test

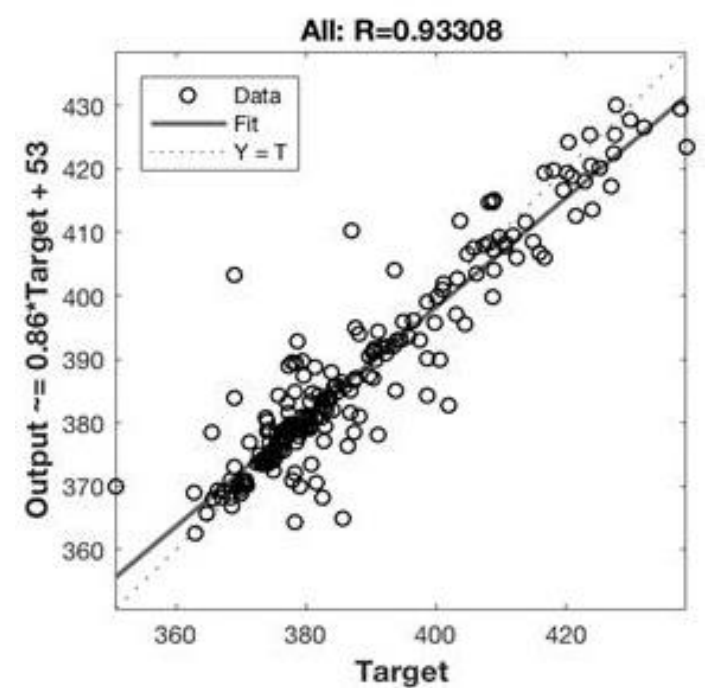

Figure 6: Correlation coefficient $\mathrm{R}$ of $\mathrm{CO}_{2}$

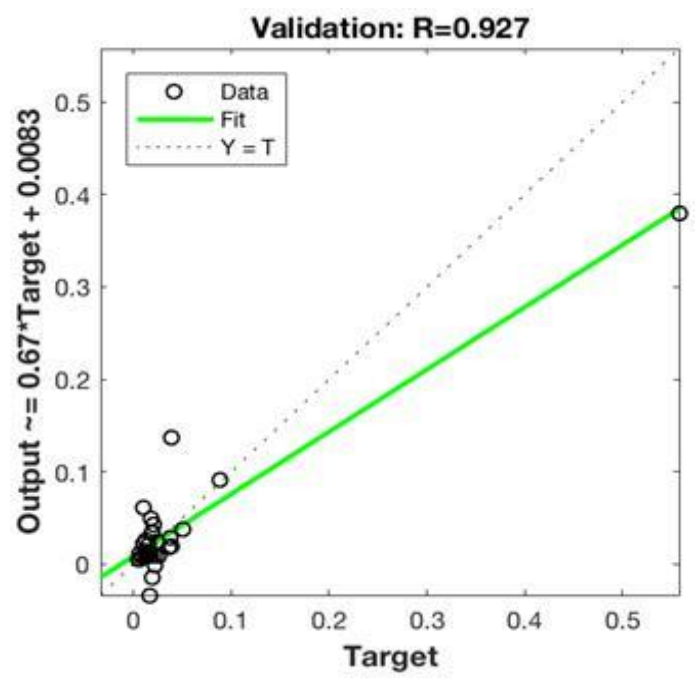

Figure 9: Correlation coefficient $\mathrm{R}$ of ammonia for test of ammonia for validation

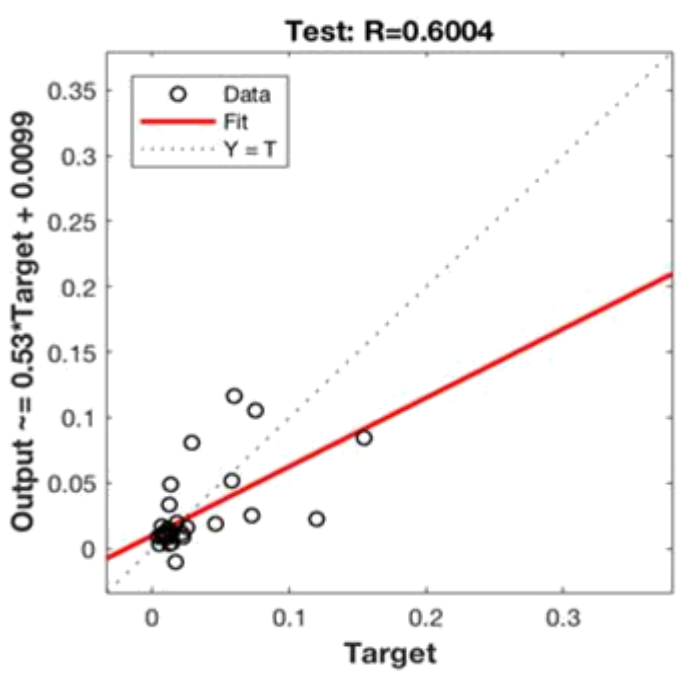

Figure 11: Correlation coefficient $\mathrm{R}$ of ammonia for all 


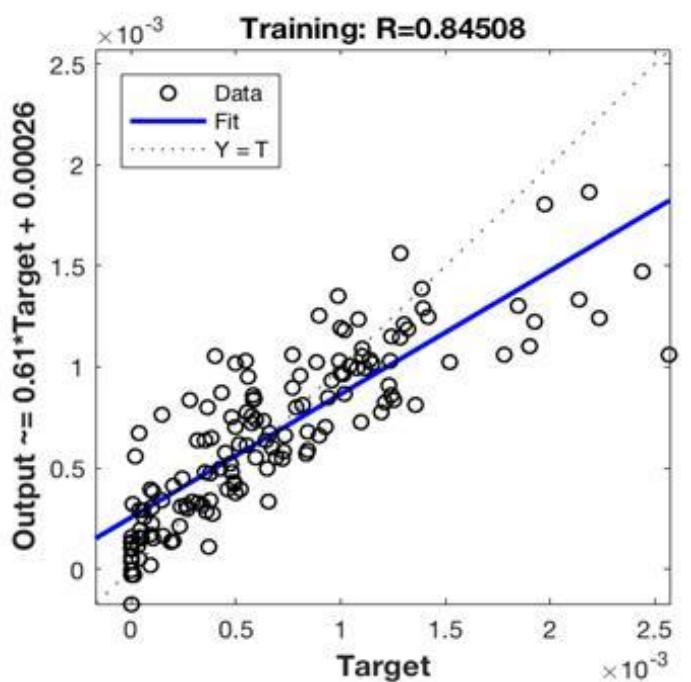

Figure 12: Correlation coefficient $\mathrm{R}$ of benzene for training

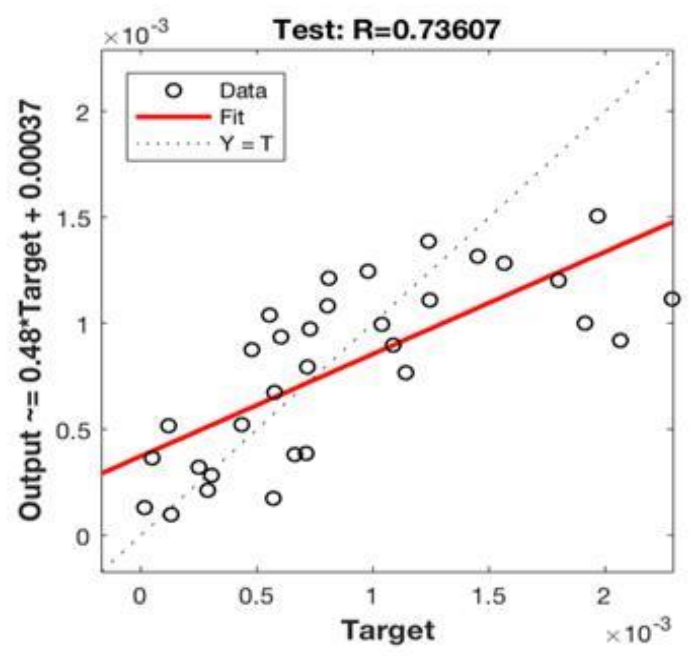

Figure 14: Correlation coefficient $\mathrm{R}$ of benzene for test

Throughout the exercise, the figure appears. It shows the network time regression versus the performance evaluation has been implemented with examination in relation depends on the value of the correlation coefficient both real as well as expected outcomes, R. Good correlation the value of the training was indicated by a dataset as well as output $\mathrm{R}$. In a regression plot, the appropriate fit shows good correlation between the predicted and targets is indicated by the solid line. The best fitting produced by the algorithm represented by dashed line. As shown, that model provide high value of correlation coefficient $\mathrm{R}$, which represents precision within measured as well as predicted values. In addition, the percentage difference was obtained between the real and predicted concentration of pollutants to show the accuracy of the ANN model by using equation. Percentage diff. $=($ predicted- real $) * 100 /$ real. The results shown in the table 2 reflect the high precision of the ANN model of prediction. Figure 16 and Figure 17 represent

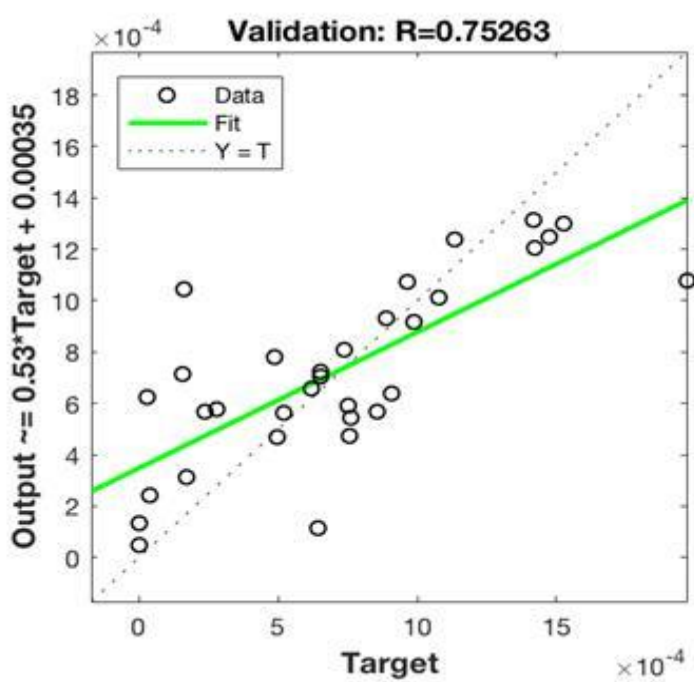

Figure 13: Correlation coefficient $\mathrm{R}$ of benzene for validation

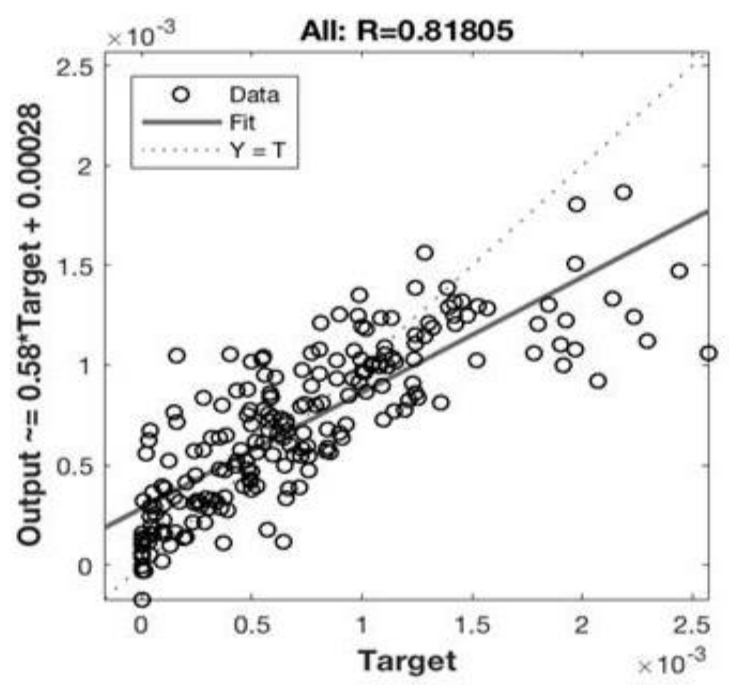

Figure 15: Correlation coefficient $\mathrm{R}$ of benzene for training

the daily concentrations of $\mathrm{CO}$ and $\mathrm{O}_{3}$ that measured by Kuwait environment public authority. In addition comparing the predicted concentrations with the standard limits set by Kuwait Environment Public as shown in Table 3 and 4, concentrations of pollutants within the study area domain shows that the pollutants concentrations results less than the standard set by KEPA, where ammonia $\left(\mathrm{NH}_{3}\right)$ was $23.94 \mathrm{ppb}$ and benzene $\left(\mathrm{C}_{6} \mathrm{H}_{6}\right)$ was $0.697 \mathrm{ppb}$.

Table 2: Percentage difference between predicted and real

\begin{tabular}{|c|c|}
\hline \multicolumn{2}{|c|}{ value } \\
\hline Pollutants & Percentage diff. \% \\
\hline $\mathrm{CO}_{2}$ & -.005 \\
\hline Ammonia & 3.572 \\
\hline Benzene & 9.3695 \\
\hline
\end{tabular}


Table 3: Ambient Air Quality Standards for Residential Areas

\begin{tabular}{|c|c|c|c|c|c|c|c|c|}
\hline \multirow[b]{2}{*}{ Pollutant } & \multicolumn{2}{|c|}{ Hour* } & \multicolumn{2}{|c|}{8 hours } & \multicolumn{2}{|c|}{ Day** } & \multicolumn{2}{|c|}{ Year } \\
\hline & Ppb & $\mu \mathrm{g} / \mathrm{m}^{3}$ & Ppb & $\mu \mathrm{g} / \mathrm{m}^{3}$ & Ppb & $\underset{3}{\mu \mathrm{g} / \mathrm{m}}$ & Ppb & $\mu \mathrm{g} / \mathrm{m}^{3}$ \\
\hline Sulfur Dioxide $\left(\mathrm{SO}_{2}\right)$ & 170 & 444 & - & - & 60 & 157 & 30 & 80 \\
\hline Nitrogen Dioxide $\left(\mathrm{NO}_{2}\right)$ & 100 & 225 & - & - & 50 & 112 & 30 & 67 \\
\hline Carbon Monoxide $(\mathrm{CO})$ & 3000 & 34000 & 10,000 & 11500 & 8000 & 9000 & - & - \\
\hline Ozone $\left(\mathrm{O}_{3}\right)$ & 80 & 157 & 60 & 120 & - & - & - & - \\
\hline Suspended particulate matter (PM-10) & - & - & - & - & - & 350 & - & 90 \\
\hline
\end{tabular}

* Average hour should not occur more than twice during the period of 30 days on the same site.

** Daily average (24 hours) should not occur more than once during the year.

\#\# Should not occur more than once per year. - Should apply in residential dominated areas that lie on the border of industrial areas.

Table 4: Criteria Pollutants

\begin{tabular}{|c|c|c|c|c|}
\hline No. & Pollutant & Averaging Time & Limit & Classification Method \\
\hline \multirow{2}{*}{1} & \multirow{2}{*}{$\mathrm{NH}_{3}$} & Hour & $800 \mathrm{ppb}$ & $\mathrm{A}$ \\
\cline { 3 - 5 } & \multirow{2}{*}{2} & 24 Hour & $144 \mathrm{ppb}$ & $\mathrm{D}$ \\
\cline { 3 - 5 } & $\mathrm{H}_{2} \mathrm{~S}$ & Hour & $100 \mathrm{ppb}$ & $\mathrm{A}$ \\
\hline 3 & & 24 Hour & $20 \mathrm{ppb}$ & $\mathrm{D}$ \\
\hline 4 & $\mathrm{C}_{6} \mathrm{H}_{6}$ & Annual & $1.6 \mathrm{ppb}$ & $\mathrm{C}$ \\
\hline
\end{tabular}

Where; A_99 ${ }^{\text {th }}$ percentile of maximum concentration over on year, B- Not exceed more than 1 time per year in the same location, C- Not to exceed, D- Not exceed more than 3 times per year in the same location.

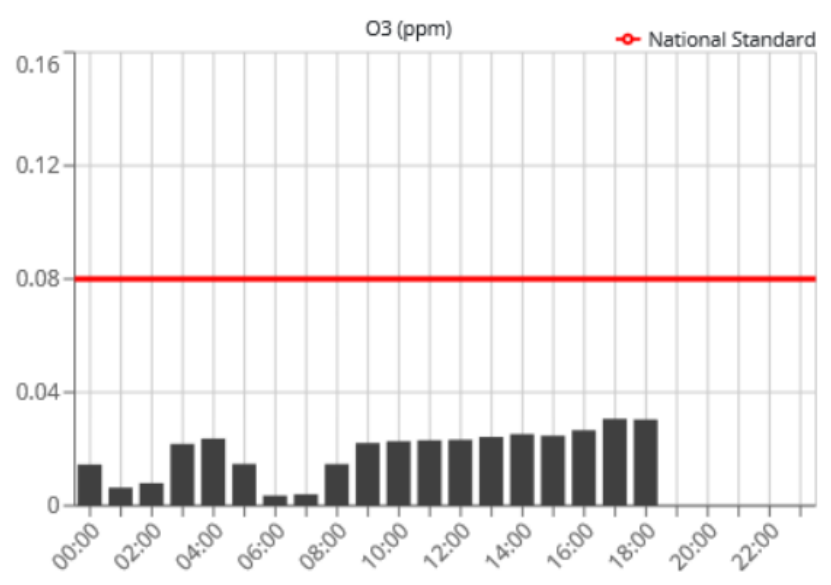

Figure 16: Concentration of $\mathrm{O}_{3}(\mathrm{ppm})$

\subsection{Optimal Back-Propagation Network Interface Setup}

For this analysis, ANN 's task for estimating trends of changes in concentration of pollutants is to use a forward propagation algorithm. The daily outside temperature, relative humidity, air pressure, wind direction and speed parameters were used as input data in the model to show the potential of the proposed ANN for modeling the pollutants concentration. There were 120 variations. Based on the minimum MAE of the training and prediction package, the real design and the parameter variance for the forward propagation model were chosen. The following approach has been adopted to simplify the forward propagation design. First, the model form has been designed with the activation function and the test scale. Third, for the maximal forward propagation model the learning and momentum levels were significant. Second, there is an optimization of the amount of neurons in the hidden layers. The

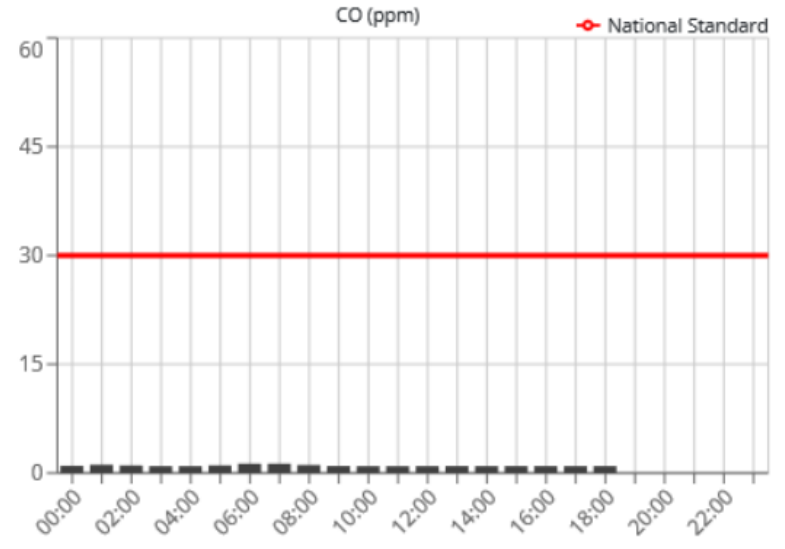

Figure 17: Concentration of CO (ppm)

initial weight was examined. Normally, as the error in the test collection rises, the testing cycle stops. The efficiency of forward propagation is influenced by all training sets (testing and predicting), irrespective of their scale. The forward propagation algorithm was required to determine the optimal values for the parameters of the neural network, such as the set percentage, number of concealed neurons, number of initial weights and learning rate and momentum rate.

Logiest networks and Gaussian functions have been equipped, evaluated and predicted with 0.1 for both learning and momentum. A forward propagation algorithm is used. The weight was 0.3 in the first place. The estimated average errors of 4 and 5 ppm respectively for the expected and preparation compartments. It was important to ensure, rather than saving from training examples, that each network knew the functional relations between the variables input and output. The data sets 
were combined allegedly for this reason and ultimately divided into three subsets of data (training, testing, and prediction). The optimal design for the forward propagation model has been learned by six different neural network architectures. The 6 architectures of the forward propagation were the following: 1) one overloaded layer; 2) two series overshadows; 3) three series overshadowed layers; 4) two parallel overshadows; 5) three parallel overshadows; and 6) two parallel layers with hop links. The optimum parameters are based on the minimal MAE as obtained from the outcomes of the preparation, testing and prediction data subsets. Table 5 shows MAEs from the six network architectures, with 4 separate evaluation percentages with $5 \%$, collected at the end of 10.000 testing epochs. The Test Collection of $5 \%$ is the following distributions with one array: [80-9-27]; e.g. 80 data testing subsets, 9 data evaluation subsets, and 27 data estimation subsets. Table 5, also indicates that the MAE forecast was somewhat higher than the MAE testing but was in general of the same magnitude suggesting sufficient network preparation. The presence of this significant prediction bias is a large gap in the network's predictive efficiency. For each of the six forward propagation architecture based on the MAEт, Table 6 presents the optimal test set. The designed architectural framework was found to be the forward propagation with two hidden parallel layers (Architecture 4), which had a $10 \%$ check scale and logistics transition (TF). Table 6, demonstrates also the influence of the MAE on training and data estimation for the same optimum layout (10 percent scale and logistic TF) of the numerical secret neurons.

It can be easily seen that the MAE of the network was much higher for the two and five numbers of neurons than those with $6,10,15$, and 20 . At 25 hidden neurons, the error increased significantly for both the training and the prediction sets. When the number of neurons exceeded 25, the average errors decreased significantly, from 9.2 to 2.8 and from 12.4 to 5.1, for both the training and the prediction sets, respectively. The neural network containing 30 hidden neurons was chosen as the best case (Table 6). In addition to the prior parameters, the initial weights, the learning rate, and the momentum rate were the other important parameters that affected the training effectiveness of the forward propagation. However, for the current case, the adjustment of the initial weight from 0.05 to 0.3 did not affect the MAE of the training and prediction networks. At the initial weight of 0.5 , the MAE increased to 8 and 11.0 for the training and prediction networks, respectively. Above the initial weight of 0.5, the network error improved (i.e., decreased) significantly (Table 5). Furthermore, varying the momentum rate and the learning rate from 0.05 to 1 , did not affect the error of the training and the prediction networks. Hence, the initial values of the learning and the momentum rates, as set by the software, were used for all training networks. Based on the results of the analyses, the optimal configuration of the ANN model was chosen, as summarized in Table 6. It can be seen from the data in these figures that the results of the model predictive ability were exceptionally good.

\subsection{Review of Sensitivity}

A sensitivity study was also performed to test the general capability of the optimum forward propagation model, by estimating the significance of each input variable individually. This analysis was done by omitting each input from the model, retraining the model, and calculating the percentage change in both the training $\left(\mathrm{MAE}_{\mathrm{T}}\right)$ and the prediction $\left(\mathrm{MAE}_{\mathrm{P}}\right)$ error functions from that of the original model.

Table 5: Effect of transfer function on mean-absolute error (MAE) for 5\% test set

\begin{tabular}{|c|c|c|c|c|c|}
\hline Model Number & $\begin{array}{l}\text { Forward propagation } \\
\text { architecture }\end{array}$ & $\begin{array}{l}\text { Logistic TF } \\
\text { MAE } \\
\text { Training } \\
\end{array}$ & Prediction & $\begin{array}{l}\text { Gaussian TF } \\
\text { MAE } \\
\text { Training } \\
\end{array}$ & Prediction \\
\hline 1 & One hidden layer & 5 & 8 & 6 & 10 \\
\hline 2 & $\begin{array}{l}\text { Two hidden layers } \\
\text { in series }\end{array}$ & 6 & 10 & 8 & 13 \\
\hline 3 & $\begin{array}{l}\text { Three hidden layers } \\
\text { in series }\end{array}$ & 7 & 6 & 10 & 12 \\
\hline 4 & $\begin{array}{l}\text { Two parallel hidden } \\
\text { layers }\end{array}$ & 5 & 8 & 7 & 10 \\
\hline 5 & $\begin{array}{l}\text { Three parallel } \\
\text { hidden Layers }\end{array}$ & 4 & 8 & 6 & 10 \\
\hline 6 & $\begin{array}{l}\text { Two parallel hidden } \\
\text { layers } \\
\text { with a jump } \\
\text { connection }\end{array}$ & 6 & 8.0 & 6.6 & 10.0 \\
\hline
\end{tabular}

Table 6: Optimum Test Set for Trained and Predictive Forward-Propagation Neural Network (BPNN) Architectures with Logistic TF

\begin{tabular}{llllll}
\multicolumn{7}{c}{ at 10,000 Training Epochs } \\
\hline $\begin{array}{l}\text { Model } \\
\text { Number }\end{array}$ & Forward propagation architecture & Training & \multicolumn{3}{c}{ Prediction } \\
\hline 1 & One hidden layer & {$[76-31-23]$} & 4.2 & {$[74-34-22]$} & 3.8 \\
2 & Two hidden layers in series & {$[80-9-41]$} & 8.5 & {$[90-16-24]$} & 10.8 \\
3 & Three hidden layers in series & {$[60-16-54]$} & 6.7 & {$[80-17-33]$} & 9.0 \\
4 & Two parallel hidden layers & {$[72-17-41]$} & 4.0 & {$[65-40-25]$} & 5.0 \\
& Three parallel hidden Layers & {$[92-34-4]$} & 4.0 & {$[93-23-14]$} & 8.5 \\
6 & Two parallel hidden layers & MAE & Optimum Test set $(\%)$ & MAE \\
\hline & with a jump connection & {$[62-31-37]$} & 6.0 & {$[79-34-17]$} & 5.0 \\
\hline
\end{tabular}


Table 7: Effect of Omitting a Specific Input from Forward-Propagation Neural Network Model on Mean-Absolute Error (MAE)

\begin{tabular}{lllll}
\hline Missing input & \% increase rate in MAET & $\mathrm{R}^{2}$ & $\%$ increase rate in MAEP & Input Strength \\
\hline Outside temperature & 67 & 0.61 & 68 & 0.25 \\
Relative humidity & 22 & 0.82 & 26 & 0.02 \\
Air pressure & 35 & 0.61 & 46 & 0.11 \\
Wind direction & 49 & 0.65 & 54 & 0.10 \\
Wind speed & 49 & 0.45 & 49 & 0.27 \\
Outside temperature and Wind direction & 35 & 0.76 & 49 & 0.58
\end{tabular}

The change in the error function is a direct measure of the predictive ability, only if the input variables are uncorrelated. Table 7 shows the percent change in the training and in the prediction model when an input variable is omitted from the neural network. The daily outside temperature, wind direction and wind speed, appear more important than the relative humidity, air pressure and wind speed parameters, for predicting the level of pollutants concentration. The least important variables are the humidity and air pressure. However, it would be incorrect to conclude that humidity and air pressure are jointly unimportant. When both outside temperature and wind direction are omitted from the neural network model, the percent change in MAE was 35 for the training and 49 for the prediction, which are lower than the percent change resulting from omitting the outside temperature, wind direction, or wind speed.

When outside air pressure was omitted from the model, the wind direction and wind speed' weight increased to compensate for the missing outside temperature, and when the wind direction and speed were omitted from the model, the air pressure weight increased to compensate for the effect. However, when the outside temperature was omitted from the model, no such compensation took place by any of the remaining variables in the model. Thus, the variable, outside temperature was more important for the training and for the prediction sets than were either wind speed or wind direction, when considered individually. It is clear from the sensitivity analysis that the outside temperature, wind direction, and wind speed have different effects on the level of pollutant concentration, and for most practical purposes, the seasonal variation (summer vs winter) would be considered more important than the rest of the parameters. It is also obvious from the analysis result that the outside temperature is associated with a much larger level of change in the output than are either the relative humidity or air pressure; for most practical purposes, the outside temperature and wind speed would be considered the most important input variables in the model because they have a pronounced effect over a large interval. Air pressure and wind direction were moderately important because they have a small effect over a large interval. Finally, the relative humidity was the least important, because they have a small effect over a small interval.

\section{Conclusion}

The current effort provides animated data and given by using the techniques ANN, Multilayer neural net-work Used for short-term development in Artificial Neural Network-based $\mathrm{CO}_{2}$, ammonia, benzene for the air-quality forecasting purposes in $\mathrm{Al}$ Ahmadi. The daily time series of these polluted concentrations, meteorological variables were collected from 2014 to 2017. Based on the present work, applying neural network provide the satisfied efficiency of prognosis of air pollution with value of $\mathrm{R}$ which represents the estimate precision. As shown values of $\mathrm{R}$ of $\mathrm{CO}_{2}$, Ammonia and Benzene respectively are $0.93308,0.81805$ and 0.923 . Which reflects a good association between the targets and expected outputs. In other wise comparing the predicted concentrations with the standard limits set by Kuwait Environment Public authority (KEPA) concentrations of pollutants within the study area domain shows very good results, where ammonia $\left(\mathrm{NH}_{3}\right)$ was $23.94 \mathrm{ppb}$ and benzene $\left(\mathrm{C}_{6} \mathrm{H}_{6}\right)$ was $0.697 \mathrm{ppb}$.

\section{Ethical issue}

Authors are aware of, and comply with, best practice in publication ethics specifically with regard to authorship (avoidance of guest authorship), dual submission, manipulation of figures, competing interests and compliance with policies on research ethics. Authors adhere to publication requirements that submitted work is original and has not been published elsewhere in any language.

\section{Competing interests}

The authors declare that there is no conflict of interest that would prejudice the impartiality of this scientific work.

\section{Authors' contribution}

All authors of this study have a complete contribution for data collection, data analyses and manuscript writing.

\section{References}

1. AAQCs "Ontario's ambient air quality criteria". https://www.ontario.ca/page/ontarios-ambient-air-quality-criteriasorted-chemical-abstracts-service-registry-number. Accessed 4 July 2017.

2. U.S. Environmental Protection Agency, "Protection of environment". $\quad$ http://www.ecfr.gov/cgi-bin/textidx? $=$ ecfr $\&$ SID $=$ bdd20ec807fda95c550270c4328e6306\&rgn=di v9\&view=text\&no de=40:6.0.1.1.6.7.1.3.38\&idno=40. Accessed 20 June 2017.

3. Kumar A, Singh BP, Punia M, Singh D, Kumar K, Jain VK. Assessment of indoor air concentrations of VOCs and their associated health risks in the library of Jawaharlal Nehru University, New Delhi. Environmental Science and Pollution Research. 2014 Feb 1;21(3):2240-8.

4. Ramadan A. Assessment of spatial variation of ambient volatile organic compound levels at a power station in Kuwait. Journal of the Air \& Waste Management Association. 2017 Nov 2;67(11):1170-9.

5. Ramadan A, Al-Rashidi M. Atmospheric monitoring at an oil field in northern Kuwait using diffusive passive samplers. International Journal of Environmental Science and Technology. 2019 Jun 6;16(6):2809-20.

6. Al-Awadhi JM. Measurement of air pollution in Kuwait City using passive samplers. Atmospheric and Climate Sciences. $2014 \mathrm{Apr}$ 2;4(02):253.

13. Gardner MW, Dorling SR. Artificial neural networks (the multilayer perceptron) - a review of applications in the atmospheric sciences. Atmospheric environment. 1998 Aug 1;32(14-15):2627-36.

14. Schalkoff, R..'Pattern recognition: statistical, structural and neural approaches". John Wiley \& Sons, Inc. New York, NY, USA., 384pp, 1992. 
15. Gardner MW, Dorling SR. Statistical surface ozone models: an improved methodology to account for non-linear behaviour. Atmospheric Environment. 2000 Jan 1;34(1):21-34.

16. Alsugair AM, Al-Qudrah AA. Artificial neural network approach for pavement maintenance. Journal of computing in civil engineering. 1998 Oct;12(4):249-55.

17. Hornik K, Stinchcombe M, White H. Multilayer feedforward networks are universal approximators. Neural networks. $1989 \mathrm{Jul}$ 1;2(5):359-66.

18. Sarle WS. Neural network frequently asked questions. ftp://ftp. sas. com/pub/neural/FAQ. html. 1998.

19. Rege MA, W. Tock R. A simple neural network for estimating emission rates of hydrogen sulfide and ammonia from single point sources. Journal of the Air \& Waste Management Association. 1996 Oct 1;46(10):953-62.

20. Haykin S. Neural networks: a comprehensive foundation. Prentice-Hall, Inc.; 2007 May 1.

21. Rumelhart DE, Hinton GE, Williams RJ. Learning internal representations by error propagation. California Univ San Diego La Jolla Inst for Cognitive Science; 1985 Sep.

22. Battiti R. First-and second-order methods for learning: between steepest descent and Newton's method. Neural computation. 1992 Mar;4(2):141-66.

23. McClelland JL, Rumelhart DE, PDP Research Group. Parallel distributed processing. Explorations in the Microstructure of Cognition. 1986;2:216-71.

24. Wasserman PD. Neural computing: theory and practice. Van Nostrand Reinhold Co.; 1989 Apr 1.

25. Comrie AC. Comparing neural networks and regression models for ozone forecasting. Journal of the Air \& Waste Management Association. 1997 Jun 1;47(6):653-63. 